\title{
Obesidad Infantil y Asma: ¿Una relación de causa y consecuencia?
}

\author{
Emilio González Jiméneza ${ }^{a}$ Judit Álvarez Ferre ${ }^{b}$
}

a Departamento de Enfermería. Facultad de Ciencias de la Salud. Universidad de Granada. Granada.

${ }^{\mathrm{b}}$ Enfermera. Hospital Universitario "San Rafael" de Granada. Granada.

Correspondencia: Emilio González Jiménez, Departamento de Enfermería, Facultad de Ciencias de la Salud, Avda. de Madrid s/n, 18071 - Granada. E-mail: emigoji@ugr.es.

Recibido el 15 de enero de 2011.

Aceptado para su publicación el 7 de abril de 2011.

\begin{abstract}
RESUMEN
El incremento de la prevalencia de obesidad en niños y en modo paralelo una incidencia cada vez mayor en los mismos de asma han motivado el planteamiento de que ambos procesos pudieran estar relacionados. Diversos estudios han demostrado un efecto de temporalidad entre estados obesogénicos y el posterior desarrollo de asma. Por otra parte, se ha descrito igualmente un efecto de dosis-respuesta, estableciéndose una relación directa entre el grado de obesidad y la severidad con la que cursa el asma en éstos sujetos. Asimismo, se ha podido comprobar cómo la pérdida de peso (por dieta) mejora y reduce los síntomas del asma. Ello parece tener su explicación en numerosos procesos orgánicos de naturaleza inmune, inflamatorios, hormonales, genéticos, dietéticos, mecánicos y de actividad física. El objetivo de este trabajo ha sido ofrecer un análisis profundo sobre los principales mecanismos implicados en la génesis del asma en el paciente pediátrico obeso.
\end{abstract}

Palabras clave: Asma, Obesidad, Niños.

\section{ABSTRACT}

Childhood Obesity and Asthma: is there a cause and effect relationship?

The increasing prevalence of obesity in children and the parallel increasing incidence of asthma has led us to propose that the two processes could be related. Several studies have shown a time effect between obesogenic states and the subsequent development of asthma. A dose response effect has also been reported, establishing a direct relationship between the degree of obesity and the severity of asthma in these subjects. It has also been demonstrated that weight loss (dieting) reduces asthma symptoms. This relationship appears to be explained by immune, inflammatory, hormonal, genetic, nutritional and mechanical factors as well as physical activity. The aim of this study is to provide a thorough analysis of the main mechanisms involved in the onset of asthma in the obese pediatric patient.

Key words. Asthma, Obesity, Children.

\section{INTRODUCCIÓN}

En la actualidad asistimos a un incremento alarmante de la prevalencia de asma entre niños y adolescentes obesos de todo el mundo ${ }^{1,2,3}$. Estudios como el desarrollado por Camargo y cols. ${ }^{4}(1999)$ han demostrado que las mujeres que ganaban peso después de los 18 años mantenían un riesgo mayor de desarrollar asma en los próximos 4 años, todo ello con independencia de su ingesta calórica y nivel de actividad física. Castro-Rodríguez y cols. ${ }^{5}$ (2001) desarrollaron el primer estudio longitudinal en población pediátrica. En él demostraron que las niñas que ganaban peso excesivamente entre los 6 y los 11 años de edad tenían siete veces más riesgo de desarrollar asma que aquellas otras en estado de normopeso. Por otra parte, estas niñas obesas presentaban una mayor respuesta broncodilatadora (volumen espiratorio forzado en el primer segundo) y una mayor variabilidad del flujo espiratorio que las chicas con normopeso. Éstas características fueron observadas exclusivamente entre las chicas. 
De acuerdo con Schaub y cols. ${ }^{6}$ (2005), la obesidad no suele tener influencia a nivel de todos los procesos alérgicos respiratorios, sino más bien, y en modo más concreto, sobre el asma y la hiperactividad bronquial.

Luego, si numerosos estudios han demostrado que la obesidad predice el desarrollo del asma (efecto de temporalidad), demostrando que a mayor grado de obesidad mayor es el efecto sobre el asma (efecto de dosis-respuesta), cabe plantear la existencia de una relación de tipo causal entre la obesidad y el asma $^{7,8}$.

Ahora bien, la explicación de esta circunstancia resulta compleja, siendo por el momento cinco los posibles procesos biológicos implicados en esta relación causal ${ }^{9}$. A continuación se describe cada unos de ellos.

\section{EFECTOS DE LA OBESIDAD SOBRE LA MECÁNICA RESPIRATORIA}

La alteración más precoz que origina la obesidad es la disminución del volumen de reserva espiratorio (VRE). Su descenso está relacionado con el volumen de masa corporal y es consecuencia del cierre precoz de las vías aéreas pequeñas existentes en las regiones más declives del pulmón por el efecto presionante del contenido abdominal sobre la posición del músculo diafragma ${ }^{10}$. Dado que esta reducción del VRE ha sido relacionada con un incremento del gradiente alvéolo-arterial de oxígeno y con la existencia de hipoxemia arterial, las alteraciones mecánicas que conducen a la disminución de este volumen serán además responsables de los desequilibrios ventilaciónperfusión ${ }^{1}$.

El descenso del volumen de reserva espiratorio (VRE), unido a un mantenimiento de la capacidad inspiratoria normal o incrementada, tendrá como consecuencia una reducción de la capacidad residual funcional (CRF), pudiendo llegar a situarse por debajo del volumen de cierre, es decir, el volumen pulmonar a partir del cual comienzan a colapsarse las primeras vías respiratorias durante una espiración lenta. El cierre prematuro de estas vías se manifestará por un aumento del shunt, y con ello un factor determinante para el descenso de la presión arterial de oxígeno con la edad. La capacidad vital (CV), el volumen espiratorio forzado en el primer segundo (FEV1) y la capacidad pulmonar total (CPT) se alteran sólo en casos de obesidad extrema. Por término general, la repercusión sobre la función respiratoria será mayor en sujetos obesos con una distribución de la grasa de predominio central ${ }^{12}$.

Otro efecto negativo de la obesidad sobre la mecánica respiratoria es el incremento del reflujo gastroesofágico, pues la obesidad está asociada a una relajación del esfínter gastroesofágico. Esta circunstancia sucede mayoritariamente entre sujetos obesos asmáticos. Esta circunstancia tiene como resultado un reflujo del ácido del esófago hacia la tráquea/vía aérea. Finalmente, el contacto directo del ácido gástrico con la vía aérea originará una broncoconstricción por microaspiración ${ }^{13}$.

\section{EFECTOS SOBRE LA RESPUESTA INMUNOLÓGICA E INFLAMATORIA}

En la actualidad se acepta que la obesidad constituye un estado proinflamatorio ${ }^{14}$. Diferentes estudios han demostrado la existencia de una relación estrecha entre obesidad y moléculas inflamatorias, entre ellas el factor de necrosis tumoral (TNF- $\alpha$ ), determinadas interleuquinas (IL) y la proteína $C$ reactiva. Se sabe que la IL-6 y el TNF- $\alpha$ se expresan en los adipocitos y se relacionan directamente con la adiposidad corporal total ${ }^{14}$. Por su parte, el TNF- $\alpha$ se encontrará igualmente aumentado entre los pacientes asmáticos, estando relacionado a su vez con la síntesis de interleuquina 4 (IL-4) e interleuquina 5 (IL-5) del tipo T helper por el epitelio bronquial ${ }^{14,15}$.

\section{INFLUENCIA DEL COMPONENTE GENÉTICO}

Se han identificado regiones específicas del genoma humano que están relacionadas tanto con el asma como con la obesidad, como por ejemplo, los cromosomas 5q, 6, 11q13 y 12q10. En el caso del cromosoma $5 q$, éste contiene los genes ADRB2 y NR3C1. El primero codifica para el receptor adrenérgico $\beta 2$, que posee influencia en la actividad del sistema nervioso simpático, y por ello importante para el control del tono de las vías aéreas y para el metabolismo basal. Por su parte, el gen NR3C1, el cual codifica para el receptor de glucocorticoides, participa en la modulación inflamatoria en procesos como el asma o la obesidad ${ }^{16}$.

En su caso, el cromosoma 6 contiene los genes del complejo principal de histocompatibilidad y del TNF- $\alpha$, los cuales influirán en la respuesta inmunitaria e inflamatoria tanto en procesos asmáticos como en la obesidad. El cromosoma $11 q 13$ contiene los genes para las proteínas desacopladoras UCP2-UPC3 y para el receptor de la inmunoglobulina $\mathrm{E}^{16}$. Las proteínas UCP2-UPC3 
influyen en el metabolismo basal, pero no en el asma. En cambio, el receptor de la inmunoglobulina E es parte de la respuesta inflamatoria de las células Th, que se incrementan en el asma, pero no en la obesidad. Por último, el cromosoma 12q contiene genes para citoquinas inflamatorias relacionadas tanto con el asma (IFN-y, LTA4H, óxido nítrico sintetasa-1) como con la obesidad (STAT6, factor de crecimiento insulinoide-1 o el CD36L1 $)^{16}$.

\section{INFLUENCIA HORMONAL Y DEL SEXO}

Datos procedentes de estudios longitudinales han evidenciado que el efecto de la obesidad sobre el asma se da más en mujeres que entre varones ${ }^{17}$. En los sujetos obesos se produce un incremento en la producción de los estrógenos, circunstancia que suele asociarse con la instauración de una menarquia precoz en las chicas y con un retraso de la pubertad entre los varones ${ }^{18}$. Este hecho parece tener su explicación en que la enzima aromatasa, responsable de convertir andrógenos en estrógenos, se encuentra presente en el tejido adiposo. Igualmente, se ha descrito que el uso de estrógenos exógenos constituye un factor de riesgo para el asma entre las mujeres ${ }^{19}$.

Así, en el estudio desarrollado por Castro-Rodríguez y cols. ${ }^{5}$ (2001) se pudo observar una mayor prevalencia de asma entre las chicas obesas que desarrollaron una menarquia temprana (antes de los once años de edad) frente a aquellas otras que la tuvieron de los once años en adelante. Estos datos sugieren que la obesidad en chicas origina alteraciones en lo referente a la síntesis y sensibilización periférica de las hormonas relacionadas con la pubertad. Esta situación, de excesiva producción o de incremento de la sensibilidad periférica frente a su acción en chicas púberes, será en última instancia la responsable de la aparición de alteraciones en el desarrollo pulmonar

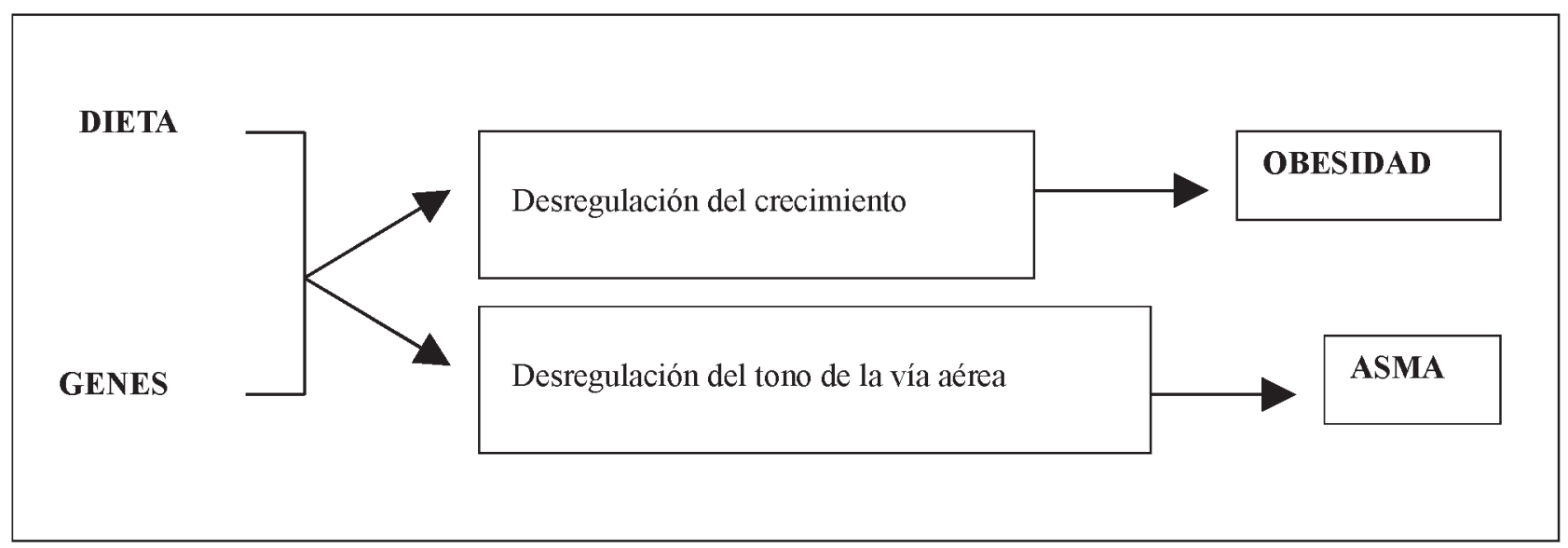

Figura 1. Interrelación entre genética y medio ambiente como base de la relación entre obesidad y asma. Adaptado de Castro-Rodríguez $\mathrm{JA}^{9}$ (2007).

y la regulación del tono de la vía aérea (entre ellas una mayor respuesta broncodilatadora).

Un tiempo después, a través de un estudio realizado en adultos por Varraso y cols. ${ }^{20}$ (2005), fue posible confirmar los planteamientos hipotéticos de estudios anteriores. Esto es, se verificó una estrecha asociación entre obesidad y asma con un predominio entre aquellas mujeres que tuvieron una menarquia temprana.

\section{OTROS FACTORES IMPLICADOS: DIETA, ACTIVIDAD FÍSICA Y "PROGRAMACIÓN FETAL"}

La dieta y la actividad física representan dos factores de enorme influencia en la obesidad y sobre el asma $^{21}$. En este sentido, determinados factores de influencia entre las mujeres embarazadas probablemente muestren también un efecto en el desarrollo fetal. Ahora bien, en la actualidad se plantea que dichos factores incidentes durante el período de vida intrauterina tendrían una repercusión a nivel del desarrollo de asma y obesidad en etapas posteriores de la vida ${ }^{22,23}$.

Del mismo modo, se ha descrito toda una variedad de factores dietéticos que guardan relación con la prevalencia del asma entre los adultos y niños. Es el caso de los antioxidantes, tales como las vitaminas $\mathrm{C}$ y $\mathrm{E}$, el caroteno, la riboflavina y la piridoxina. Todos ellos parecen tener efectos relevantes incrementando la función y respuesta 
inmunológica, reduciendo los síntomas del asma, así como mejorando la función pulmonar ${ }^{24}$.

En el estudio de Romieu y cols. ${ }^{25}$ (2006), se puso de manifiesto cómo aquellas mujeres adultas que consumían frutas y verduras (tomates, zanahorias y vegetales de hoja) tenían una menor prevalencia de asma. Por otra parte, un mayor consumo de ácidos grasos trans entre la población pediátrica se relaciona igualmente con una reducción de la prevalencia del asma (Weiland y cols., 1999) ${ }^{26}$. Otros trabajos, como los desarrollados por Barker y cols. ${ }^{27}$ (1991) y Svanes y cols. ${ }^{28}$ (1998) encontraron una estrecha relación entre peso al nacimiento (con independencia de la edad gestacional) y el riesgo de asma. Luego, tanto el bajo peso al nacer como el alto se correlacionaron con un mayor riesgo de incidencia de asma. Todo ello parece ser indicativo de la existencia y realidad de una "programación fetal", programación que estaría implicada en el desarrollo de obesidad y asma en etapas ulteriores de la vida. En la figura número 1 se muestra la interrelación existente entre genética y medioambiente como base para la relación entre obesidad y asma ${ }^{9}$.

\section{CONCLUSIONES}

La compleja interrelación entre obesidad y asma constituye un claro ejemplo de cómo los genes y el ambiente pueden interactuar en la génesis de ambas entidades ${ }^{29}$.

La obesidad por medio de procesos inflamatorios, ayudados a su vez por cambios en los estilos de vida, puede ser el desencadenante de la aparición de síntomas asmáticos ${ }^{30}$. La interacción entre dieta y genes puede causar alteraciones en el patrón de crecimiento corporal, originando obesidad y a su vez perturbando el tono de la vía aérea (originando asma).

El personal sanitario en su conjunto debe estar familiarizado con todas las manifestaciones respiratorias de la obesidad, en tanto que se prevé un incremento en el número de consultas relacionadas con ella en los años venideros ${ }^{31}$. Del mimo modo, sería deseable y necesario poner en marcha estudios que profundizasen y aclarasen los entresijos aún por conocer de la relación existente entre la obesidad y las alteraciones respiratorias como el asma. Además, resultará igualmente necesario establecer colaboraciones entre los profesionales de las distintas especialidades
(Endocrinología, Cardiología, etc.), todo ello orientado a poner en marcha programas de acción y prevención coordinados.

\section{AGRADECIMIENTOS}

Los autores de este trabajo muestran su más sincero agradecimiento al Departamento de Enfermería de la Universidad de Granada por todas las facilidades brindadas en la realización de dicho trabajo de revisión.

\section{BIBLIOGRAFIA}

1. World Health Organization. Obesity: preventing and managing the global epidemic. WHO Technical Report Series 894. Geneva: WHO; 2000.

2. Chinn S. Obesity and asthma. Paediatr Respir Rev. 2006; 7:223-28.

3. Pérez de Llano LA. Efectos de la obesidad sobre el aparato respiratorio. Pneuma. 2007; 7:19-26.

4. Zammit $\mathrm{C}$, Liddicoat $\mathrm{H}$, Moonsie I, Makker H. Obesity and respiratory diseases. Int J Gen Med. 2010; 20(3):335-43.

5. Raviv S, Dixon AE, Kalhan R, Shade D, Smith LJ. Effect of Obesity on Asthma Phenotype is Dependent upon Asthma Severity. J Asthma. 2011; 48(1):98-104.

6. Schaub B, von Mutius E. Obesity and asthma, what are the links?. Curr Opin Allergy Clin Immunol. 2005; 5:185-93.

7. Von Mutius E, Schwartz J, Neas LM, Dockery D, Weiss ST. Relation of body mass index to asthma and atopy in children: the National Health and Nutrition Examination Study III. Thorax. 2001; 56:835-38.

8. Chinn S, Rona RJ: Can the increase in body mass index explain the rising tend in asthma in children? Thorax. 2001; 56:845-50.

9. Castro-Rodríguez JA. Relación entre obesidad y asma. Arch Bronconeumol. 2007; 43(3):171-75.

10. Sood A. Sex Differences: Implications for the ObesityAsthma Association. Exerc Sport Sci Rev. 2010; 39(1):4856.

11. Dogra S, Baker J, Ardern Cl. Role of age at asthma diagnosis in the asthma-obesity relationship. Can Respir J. 2010; 17 (5):97-101.

12. De Groot EP, Duiverman EJ, Brand PL. Comorbidities of asthma during childhood: possibly important, yet poorly studied. Eur Respir J. 2010; 36(3):671-78.

13. Mckenzie SM, Bush A. Difficult asthma in children. Arch Dis Child. 2003; 88:168-179.

14. Cottrell L, Neal WA, Ice C, Perez MK, Piedimonte G. Metabolic Abnormalities in Children with Asthma. Am J Respir Crit Care Med. 2011; 183(4):441-8.

15. Dixon $A E$, Holguin $F$, Sood $A$, Salome $C M$, Pratley RE, Beuther DA, et al. An official American Thoracic Society Workshop report: obesity and asthma. Proc Am Thorac Soc. 2010; 7(5):325-35.

16. Beuther DA. Recent insight into obesity and asthma. Curr Opin Pulm Med. 2010; 16(1):64-70.

17. Lugogo NL, kraft M, Dixon AE. Does obesity produce a distinct asthma phenotype? J Appl Physiol. 2010; 108(3):729-34.

18. Delgado J, Barranco P, Quince S. Obesity and asthma. J Investig Allergol Clin Immunol. 2008; 18(6):420-25.

19. Shore SA. Obesity and asthma: possible mechanisms. J Allergy Clin Immunol. 2008; 121(5):1087-93. 
20. Varraso R, Siroux V, Maccario J, Pin I, Kauffmann F. Asthma severity is associated with body mass index and early menarche in women. Am J Respir Crit Care Med. 2005; 171:334-39.

21. Willers SM, Wijga AH, Brunekreef B, Scholtens S, Postma DS, Kerkhof M, et al. Childhood diet and asthma and atopy at 8 years of age: the PIAMA birth cohort study. Eur Respir J. 2010; 25 [Epub ahead of print].

22. Hakala K, Steinus-Aarnalia B, Sovijarvi A. Effects of weight loss on peak flow variability, airways obstruction and lung volumes in obese patients with asthma. Chest. 2000; 118:1315-321.

23. Stenius-Aarnalia B, Poussa T, Kvarnstrom J, Gronlund EL, et al. Immediate and long term effects of weight reduction in obese people with asthma: randomized controlled study. Brit Med J. 2000; 320(7238):827-32.

24. Olivares-Corichi IM, Martín A, Sierra MP, Mendoza Atencio RS, Hicks JJ. Perspectivas del uso de antioxidantes como coadyuvantes en el tratamiento del asma. Rev Inst Nal Enf Resp. 2005; 18(2):154-61.

25. Romieu I, Varraso R, Avenel V, Leynaert B, Kauffmann F, Clavel- Chapelon F. Fruit and vegetable intakes and asthma in the E3N study. Thorax. 2006; 61:209-15.

26. Minas M, Papaioannou AI, Tsaroucha A, Daniil Z, Hatzoglou $\mathrm{C}$, Sgantzos $\mathrm{M}$, et al. Body composition in severe refractory asthma: comparison with COPD patients and healthy smokers. PLoS One. 2010; 5(10): e13233.

27. Budweiser S, Meyer K, Jörres RA, Heinemann F, Wild PJ, Pfeifer M. Nutritional depletion and its relationship to respiratory impairment in patients with chronic respiratory failure due to COPD or restrictive thoracic diseases. Eur $\mathrm{J}$ Clin Nutr. 2008; 62(3):436-43.

28. Vestbo J, Prescott E, Almdal T, Dahl M, Nordestgaard BG, Andersen, et al. TBody mass, fat-free body mass, and prognosis in patients with chronic obstructive pulmonary disease from a random population sample: findings from the Copenhagen City Heart Study. Am J Respir Crit Care Med. 2006; 173(1):79-83.

29. Hitzl AP, Jörres RA, Heinemann F, Pfeifer M, Budweiser S. Nutritional status in patients with chronic respiratory failure receiving home mechanical ventilation: impact on survival. Clin Nutr. 2010; 29(1):65-71.

30. Guerra S, Sherrill DL, Bobadilla A, Martinez FD, Barbee RA. The relation of body mass index to asthma, chronic bronchitis, and emphysema. Chest. 2002; 122(4):1256263.

31. Weiss ST, Shore S. Obesity and asthma: directions for research. Am J Respir Crit Care Med. 2004; 169(8):96368. 